\title{
The Dorsal Attention Network Mediates Orienting toward Behaviorally Relevant Stimuli in Spatial Neglect
}

\author{
Radek Ptak ${ }^{1,2}$ and Armin Schnider ${ }^{1,2}$ \\ ${ }^{1}$ Division of Neurorehabilitation, Geneva University Hospitals, and 2Faculty of Medicine, University of Geneva, 1211 Geneva 14, Switzerland
}

Experimental neurophysiology and functional neuroimaging have identified a dorsal attention network that encodes neural signals related to the behavioral significance of a stimulus. The core anatomical areas of this network are the frontal eye fields and the posterior parietal cortex, which are interconnected by the superior longitudinal fasciculus. Here, we show that damage or disconnection of this network predicts the extent to which task-relevant stimuli capture attention of human stroke patients with spatial neglect. Healthy volunteers, right-hemisphere-damaged control participants, and patients with left neglect reacted to peripheral targets defined by their color, which were preceded by a brief distracter stimulus. The position of the distracter and its relevance for the current trial were independently varied. In neglect patients with damage including the frontal eye fields and the superior longitudinal fasciculus, ipsilesional distracters impaired orienting into contralesional space regardless of their relevance for the current task. In contrast, patients with sparing of these regions were only impaired when distracters were task-relevant. These findings indicate that the dorsal attention network controls spatial orienting by modulating the saliency of distracter stimuli according to current action goals.

\section{Introduction}

The capacity to detect and orient toward stimuli in surrounding space is mediated by a cortical network distributed over the parietal and prefrontal association cortices (Gitelman et al., 1999; Corbetta and Shulman, 2002). Orienting attention in space is driven by current action goals of the individual or by physical characteristics of the stimulus (Egeth and Yantis, 1997). However, these processes are not fully independent, as even seemingly automatic capture of attention is modulated by task relevance (Yantis and Jonides, 1990; Folk et al., 1992). Two fundamental questions therefore are: where in the attention network do representations of stimulus relevance form and how do they interact to produce shifts of attention toward stimuli that match current action goals. Neurophysiological studies have revealed that activity of neurons in the posterior parietal cortex (PPC) and the prefrontal cortex of the monkey is biased by task relevance. Thus, PPC neurons react stronger to a stimulus previously defined as an action target than to a neutral stimulus (Gottlieb et al., 1998; Constantinidis and Steinmetz, 2001). Similarly, neurons in the frontal eye field (FEF), a cortical area with a decisive role in saccade programming, show enhanced responses to a visual stimulus when it is the saccade target (Bruce and Goldberg, 1985; Bichot and Schall, 1999; Schall and Thompson, 1999). Consistent

Received May 28, 2010; revised June 25, 2010; accepted July 1, 2010.

This study was supported by the Swiss National Science Foundation (Grant 320000-113438) and the de Reuter Foundation (Grant 458). We thank K. Gutbrod for technical advice regarding lesion analysis and L. Golay for testing several healthy participants.

Correspondence should be addressed to Radek Ptak, Division of Neurorehabilitation, Geneva University Hospitals and Faculty of Medicine, University of Geneva, 26, Avenue de Beau-Séjour, 1211 Geneva 14, Switzerland. E-mail: radek.ptak@hcuge.ch.

DOI:10.1523/JNEUROSCI.2722-10.2010

Copyright $\odot 2010$ the authors $\quad 0270-6474 / 10 / 3012557-09 \$ 15.00 / 0$ with these findings, attending to task-relevant stimulus features strongly activates the PPC and the FEF (Corbetta et al., 2000; Hopfinger et al., 2000; Indovina and Macaluso, 2007). As the FEF responds extremely quickly to sensory stimulation, the propagation of task-related modulation of activity through the attention network may have its origin here (Fecteau and Munoz, 2006; Buschman and Miller, 2007; Kirchner et al., 2009).

Extensive right parietal, temporal, or frontal damage may lead to spatial neglect, a disorder characterized by the failure to detect and respond to stimuli presented in contralesional space (Heilman et al., 1993; Halligan et al., 2003). Because of their strong attentional bias toward stimuli in the preserved visual field and their difficulty reorienting attention contralesionally (Posner et al., 1984; Morrow and Ratcliff, 1988), neglect patients are ideal participants for the study of task-related modulations of attention. However, whereas in some studies only task-relevant distracters captured attention of neglect patients (Ptak and Golay, 2006; Ptak and Schnider, 2006), other studies observed an unselective bias favoring ipsilesional stimuli regardless of their relevance for the current task (Gainotti et al., 1991; Snow and Mattingley, 2005).

Here, we sought to determine the source of this discrepancy by examining whether damage to specific brain structures predicts the capacity of neglect patients to reorient attention following presentation of a distracter that shares task-relevant properties with the target. Patients reacted to colored rectangles appearing left or right of fixation following a brief peripheral cue with a color that was either task relevant or irrelevant. We found that neglect patients with preserved FEF and its posterior connections exhibited a selective bias favoring task-relevant ipsilesional cues, whereas when these regions were damaged attention was biased toward all stimuli, regardless of their relevance. 
Table 1. Demographic characteristics and performance in clinical neglect tests (mean \pm SEM)

\begin{tabular}{|c|c|c|c|c|c|c|c|}
\hline & Age & $\begin{array}{l}\text { Time post } \\
\text { (days) }\end{array}$ & $\begin{array}{l}\text { Bells cancellation } \\
\text { (left omissions) }\end{array}$ & $\begin{array}{l}\text { T cancellation } \\
\text { (left omissions) }\end{array}$ & $\begin{array}{l}\text { Line bisection } \\
\text { (\% right bias) }\end{array}$ & $\begin{array}{l}\text { Sentence copying } \\
\text { (words missed) }\end{array}$ & Drawing \\
\hline Relevance neglect & $68.1( \pm 4.6)$ & $54.9( \pm 10.1)$ & $10.7( \pm 1.5)$ & $17.3( \pm 2.9)$ & $10.5( \pm 3.0)$ & $3.9( \pm 1.8)$ & $0.7( \pm 0.3)$ \\
\hline Nonrelevance neglect & $62.7( \pm 5.1)$ & $37.8( \pm 7.2)$ & $9.9( \pm 1.8)$ & $17.5( \pm 3.4)$ & $10.6( \pm 2.6)$ & $4.4( \pm 2.5)$ & $0.9( \pm 0.3)$ \\
\hline RH controls & $66.8( \pm 2.2)$ & $57.8( \pm 13.4)$ & $2.1( \pm 0.8)$ & $0.6( \pm 0.3)$ & $4.0( \pm 1.1)$ & $0( \pm 0)$ & $2( \pm 0)$ \\
\hline Healthy controls & $63.5( \pm 2.7)$ & - & - & - & - & - & - \\
\hline
\end{tabular}

Drawing performance was scored as follows: $0=$ flagrant omissions on the left side; $1=$ distorted left side; $2=$ intact.

\section{Materials and Methods}

Participants. Twenty patients with left spatial neglect (13 females), 10 right-hemisphere- $(\mathrm{RH})$ damaged control patients without neglect $(4$ females), and 10 healthy controls ( 6 females) participated to the study. Approval was obtained from the ethical committee of the University Hospitals Geneva (Geneva, Switzerland) and all participants gave written consent. All patients were examined while hospitalized at the Division of Neurorehabilitation of the University Hospital Geneva following a firstever stroke or hemorrhage.

One of the most consistent findings of spatial cueing studies examining neglect patients is an impairment of attentional reorienting toward contralesional targets following an ipsilesional distracter stimulus (the disengagement deficit) (Posner et al., 1984; Morrow and Ratcliff, 1988; Losier and Klein, 2001). We previously observed that the disengagement deficit was much stronger when cues shared a task-relevant property with the target (Ptak and Schnider, 2006). To identify the cerebral structures that were critical for the modulation of the disengagement deficit by task relevance, we examined a large group of neglect patients and then differentiated those patients who showed task-relevant modulation of attention from patients who did not. These two subgroups of neglect patients were distinguished by calculating the difference between reaction times to left targets following right relevant cues and right irrelevant cues for each participant and submitting these difference scores to a $k$-means cluster analysis. Cluster analysis is an exploratory data analysis technique that attempts to find groups in data that are characterized by high internal similarity while being maximally dissimilar to other groups. The analysis classified a subgroup of neglect patients with large reaction time differences as belonging to cluster 1 (neglect patients 1-10; hereafter termed the relevance-neglect group) and all other participants including neglect patients 11-20 (hereafter termed the nonrelevance neglect group) as belonging to cluster 2 . This classification was highly significant (ANOVA, $\left.F_{(1,38)}=99.67, p<0.00001\right)$. In addition, all patients of the relevance-neglect group, but none of the nonrelevance group, had significantly increased reaction times to left targets following relevant than irrelevant cues ( $t$ test on individual patient data; all $p<0.05$ ).

Clinical neglect tests were performed in the same week as the experimental task. Table 1 shows demographic data and the results of clinical testing of neglect and control patients. All patients had preserved visual fields for the central $\sim 20^{\circ}$ as assessed with computerized perimetry testing (white dot presented on black background at positions ranging between -25 and +25 degrees) and/or clinical confrontation. All neglect patients manifested behavioral signs of left unawareness (e.g., failure to notice objects or persons placed on their left, difficulties with dressing or grooming) and lateralized failures in the following neglect tests: Bells cancellation (Gauthier et al., 1989), cancellation of inverted Ts (Ptak et al., 2007), line bisection (Schenkenberg et al., 1980), sentence copying (Wilson et al., 1987), and copying a landscape. Participants had similar age (ANOVA across all four groups, $F_{(3,36)}=0.44$ ), and the three patient groups had comparable time since injury $\left(F_{(2,27)}=0.45\right)$. The two neglect groups had statistically indistinguishable performance in all neglect tests (Mann-Whitney test, all $p>0.25$ ), but scored significantly worse compared with RH controls in all except the sentence-copying test (all $p<0.02$ ).

Stimuli and procedure. Participants were asked to react to a colored rectangle appearing left or right of fixation following a briefly presented, peripheral cue (Fig. 1). Stimuli were rectangles subtending $4 \times 2^{\circ}$ at 60 $\mathrm{cm}$ viewing distance, filled red, green, blue, or yellow and presented on neutral gray background, with their inner border $1.5^{\circ}$ from fixation. The

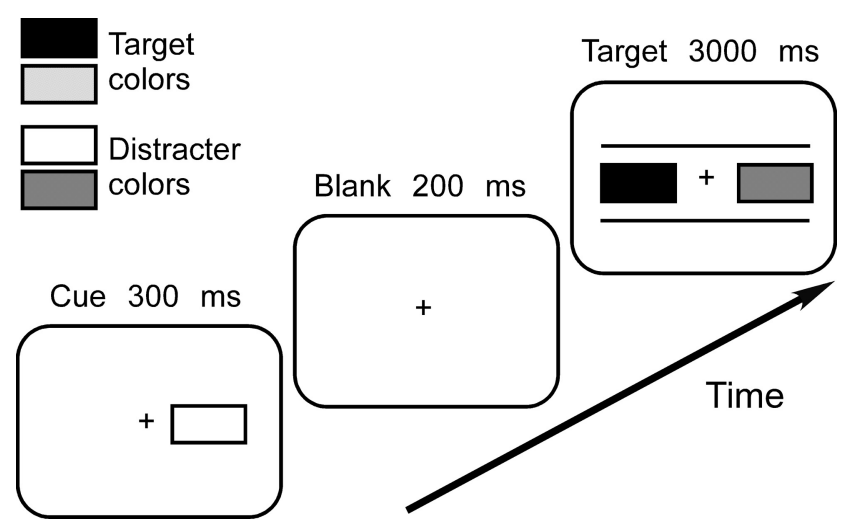

Figure 1. Stimuli and timing of the experiment. For every participant, two target and two distracter colors (represented with different shades of grey) were selected randomly. Participants were instructed to ignore the peripheral cue and to react when the target display contained a target color. The cue appeared on the same side as the upcoming target (valid cue) or on the opposite side (invalid cue) and either had the same color as the target (relevant identical cue), the other target color (relevant different cue), or a distracter color (irrelevant cue). Cue validity and relevance were unpredictive of target presence or position. The figure shows a left invalid-irrelevant trial.

target display consisted of two rectangles placed between two white horizontal lines that were irrelevant for the task, but were presented to clearly differentiate the target display from the cue display. The cue was a single rectangle presented left or right of fixation. For each participant, two of four colors (e.g., red and blue) were randomly defined as target colors whereas the other colors were distracters. The cue either had a taskrelevant and identical color as the target (e.g., red cue, red target), a task-relevant but different color than the target (e.g., blue cue, red target), or a task-irrelevant color (e.g., green cue, red target). In addition, it appeared at the same side as the upcoming target (valid cue) or at the opposite side (invalid cue). Relevant/irrelevant cues and valid/invalid cues were equally likely, and the occurrence of a target and its position could not be inferred from the presence or position of a particular cue. The most sensible strategy was therefore to ignore the cue and to focus attention uniquely on the target display.

On every trial, participants first fixated a white central cross for 2000 $\mathrm{ms}$. The cue was presented for $300 \mathrm{~ms}$, followed by a blank screen for 200 $\mathrm{ms}$. The target display then appeared and remained on screen for $3000 \mathrm{~ms}$ or until a button press occurred. On target-present trials, the target display contained one color target on the left or right side paired with a color distracter. On target-absent trials, two color distracters were shown. Participants were asked to press down the space key when they detected the target and to withhold reaction on target-absent trials. Fixation was controlled visually during a practice run and checked periodically during the experimental runs. Target position (left, right), cue relevance (relevant identical, relevant different, irrelevant) and validity (valid, invalid) were orthogonally varied in blocks of 72 trials containing 48 target-present and 24 target-absent trials.

Lesion mapping. All except one patient of the RH-control group underwent structural magnetic resonance imaging (MRI), including T1and T2-weighted acquisitions with a between-slice resolution of $4 \mathrm{~mm}$, on a 1.5 T MRI scanner (Siemens Vision). The median time between stroke and imaging was $10 \mathrm{~d}$. For each patient, the area of damage [volume of interest (VOI)] was delineated directly on an axial T2-weighted 
MRI scan using a graphics tablet and MRIcron (Rorden et al., 2007). If diffusion-weighted images were available, they were used to aid lesion mapping. Each patient MRI including the lesion VOI was then normalized to standard space using SPM5 (www.fil.ion.ucl.ac.uk/spm). To minimize normalization artifacts due to contribution of abnormal tissue, the lesion was excluded from the computation of the transformation matrix using a lesion mask. Normalized lesion VOIs were projected onto the T1-weighted template MRI scan from the Montreal Neurological Institute (MNI; http://www.bic.mni.mcgill.ca), and overlaps and voxel-based comparisons were performed using MRIcron. All voxel coordinates are indicated in MNI space.

The most direct assessment of the relation between a behavioral measure and anatomy is provided by voxel-based lesion-symptom mapping (VLSM). However, previous studies of neglect anatomy almost exclusively applied lesion subtraction methods; to allow comparisons of our results to those of previous studies we therefore first used this behaviordefined approach (Bates et al., 2003) and performed conventional subtraction analyses of our data. Gray matter and white matter involvement was determined by segmenting gray and white matter of the MNI template brain using SPM5 and then calculating the overlap with lesion VOIs. To identify the overlap of individual lesion VOIs with different Brodmann areas, we segmented VOIs using the Brodmann template distributed with MRIcron. Finally, segmentation of VOIs was also performed using selected white-matter fiber tracts from the Johns Hopkins University (Baltimore, Maryland) group-averaged atlas based on deterministic diffusion-tensor imaging tractography (Mori et al., 2008). The overlap of individual VOIs with Brodmann areas and fiber tracts was determined on a voxel-by-voxel basis, and the segmented VOIs were then compared between patient groups.

These comparisons were followed up with VLSM analyses. Since the primary objective of this study was to identify the brain regions that predict the degree to which ipsilesional task-relevant or irrelevant cues capture attention in neglect patients, the VLSM analyses were performed using the data of the two neglect groups. MRIcron offers two VLSM methods to study the relation between a behavioral measure and anatomy. The nonparametric Liebermeister test is performed on binomial data and thus requires patients to be assigned to two different groups based on a behavioral measure. The more conservative, nonparametric Brunner-Munzel test identifies brain regions that are critical for performance using a continuous behavioral measure. As we sought for converging evidence, we used both methods to analyze the lesion data of neglect patients. To control for multiple comparisons, family-wise error rates (FWE) were computed using repeated permutation tests, which provide a more powerful correction for multiple comparisons than the Bonferroni correction (Rorden et al., 2007). Only voxels damaged in at least $20 \%$ of all patients were included in the VLSM analyses.

\section{Results}

\section{Behavioral results}

Target misses were rare in healthy participants (mean omission rate, $0.4 \%$ ) and $\mathrm{RH}$-control patients $(1.3 \%)$. These omission rates were close to ceiling and were therefore not analyzed further. Both neglect groups missed significantly more targets presented in the left compared with the right hemifield (relevance group: $5.9 \%$ vs $2.1 \%$; Wilcoxon test, $p<0.05$; nonrelevance group: $5.7 \%$ vs $2.0 \%, p<0.05)$. Similarly to target misses, falsepositive responses (i.e., reactions to nontarget stimuli) were rare in healthy $(1.3 \%)$ and RH controls $(1.4 \%)$. Both neglect groups produced more false-positive responses following relevant (relevance group, 9.1\%; nonrelevance group, 7.6\%) than irrelevant cues (relevance group, 1.7\%; nonrelevance group, $0.4 \%$ ), but their falsepositive rates were comparable (Mann-Whitney test, $p=0.31$ ).

Figure 2 shows reaction time (RT) data of the four participating groups. In an initial analysis, these data were submitted to a repeated-measures ANOVA with factors group (healthy controls, $\mathrm{RH}$ controls, relevance neglect, nonrelevance neglect), target position (left, right hemifield), cue validity (valid, invalid), and cue
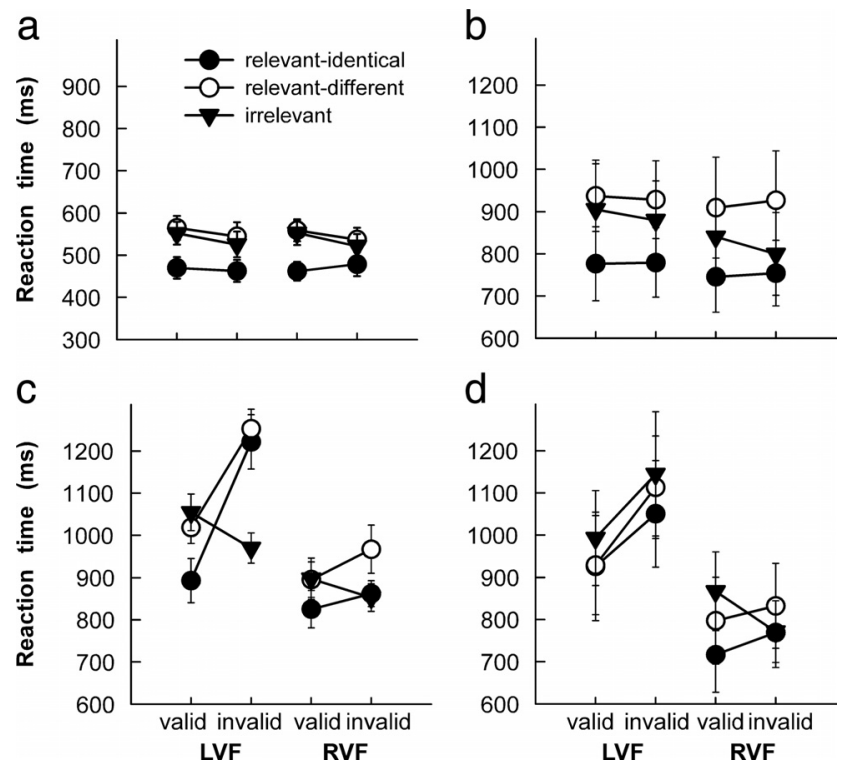

Figure 2. $\quad \boldsymbol{a}-\boldsymbol{d}$, Reaction-time data of healthy controls $(\boldsymbol{a})$, right-hemisphere-damaged controls $(\boldsymbol{b})$, neglect patients with a relevance effect $(\boldsymbol{c})$, and neglect patients without a relevance effect $(\boldsymbol{d})$. Note that different scales were used to represent data of healthy participants and patients. LVF, Left visual field; RVF, right visual field. Error bars show SEM.

relevance (relevant identical, relevant different, irrelevant). This analysis revealed significant main effects of group $\left(F_{(3,36)}=8.47\right.$, $p<0.001)$, target position $\left(F_{(1,36)}=30.64, p<0.0001\right)$, cue validity $\left(F_{(1,36)}=5.59, p<0.05\right)$, and cue relevance $\left(F_{(2,72)}=\right.$ $49.31, p<0.0001)$. A number of two-way interactions were also significant: group $\times$ target position $\left(F_{(3,36)}=7.34, p<0.0001\right)$, group $\times$ cue validity $\left(F_{(3,36)}=3.29, p<0.05\right)$, group $\times$ cue relevance $\left(F_{(6,72)}=6.21, p<0.0001\right)$, target position $\times$ cue validity $\left(F_{(1,36)}=12.55, p<0.01\right)$, and cue validity $\times$ cue relevance $\left(F_{(2,72)}=38.78, p<0.0001\right)$. The three-way interactions between group $\times$ target position $\times$ cue validity $\left(F_{(3,36)}=4.94, p<0.01\right)$ and group $\times$ cue validity $\times$ cue relevance $\left(F_{(6,72)}=11.48, p<\right.$ $0.0001)$ were also significant. Finally, the four-way interaction group $\times$ target position $\times$ cue validity $\times$ cue relevance was significant $\left(F_{(6,72)}=5.22, p<0.001\right)$.

Given the significant main effect of group and the high number of significant interactions including the factor group, we decided to follow up these results with separate analyses of the data of each group, focusing on the factors target position, cue validity, and cue relevance. The highest-order significant effects were followed up with post hoc Scheffé tests. The data of healthy participants (Fig. 2a) and of RH control participants (Fig. $2 b$ ) were characterized by a significant main effect of relevance (healthy, $F_{(2,18)}=34.94, p<0.0001 ; \mathrm{RH}$ controls, $F_{(2,18)}=24.26, p<$ $0.0001)$. In both groups and independently of cue validity, relevant-identical cues facilitated target detection relative to relevant-different and irrelevant cues (all $p<0.01$ ), although the latter two resulted in indistinguishable performance. No other effect or interaction reached significance.

In contrast, performance of the relevance-neglect group (Fig. $2 c$ ) depended on target position $\left(F_{(1,9)}=24.05, p<0.001\right)$, validity of the cue $\left(F_{(1,9)}=11.48, p<0.01\right)$, and cue relevance $\left(F_{(2,18)}=10.88, p<0.001\right)$, as well as the interaction position $X$ validity $\left(F_{(1,9)}=8.43, p<0.05\right)$ and validity $\times$ relevance $\left(F_{(2,18)}=\right.$ 32.03, $p<0.0001)$. Importantly, the three-way interaction was significant $\left(F_{(2,18)}=8.96, p<0.01\right)$, reflecting two important effects. First, patients detected left targets faster following valid 
cues than invalid cues only if cues were relevant identical (RTs following valid vs invalid cues, $893 \mathrm{~ms}$ vs $1222 \mathrm{~ms}, p<$ $0.001)$ or relevant different (1019 ms vs $1253 \mathrm{~ms}, p<0.05)$, but not when they were irrelevant (1055 ms vs $970 \mathrm{~ms})$. In other words, this group showed a disengagement deficit with relevant cues only. Second, detection of invalidly cued left targets was slower following relevant identical $(p<0.01)$ and relevant different $(p<0.01)$ than irrelevant cues. Similarly, the nonrelevance neglect group reacted slower to left than right targets $\left(F_{(1,9)}=\right.$ 10.98, $p<0.01$ ) (Fig. $2 d$ ) and following irrelevant than relevant cues $\left(F_{(2,18)}=\right.$ 8.53, $p<0.01)$. Importantly, the data of this group also exhibited a significant interaction of target position $\times$ validity $\left(F_{(1,9)}=6.51, p<0.01\right)$, reflecting the fact that attentional reorienting after presentation of invalid cues was only impaired for left targets (RTs to left targets following valid vs invalid cues, 949 ms vs $1103 \mathrm{~ms}, p<0.05$; right targets, $794 \mathrm{~ms}$ vs $791 \mathrm{~ms}$ ). This is the critical finding indicating a deficit of attentional disengagement from ipsilesional cues (Posner et al., 1984; Morrow and Ratcliff, 1988; Losier and Klein, 2001). In contrast to the relevance-neglect group, however, the three-way interaction was not significant $\left(F_{(2,18)}=2.13, p=0.15\right)$, indicating that the deficit of attentional reorienting toward contralesional targets was similar for relevant and irrelevant cues. Thus, both neglect groups were impaired in reorienting attention to a contralesional target following an ipsilesional cue; however, although one group only failed to reorient attention following relevant cues, attentional reorienting of the other group was not influenced by cue relevance.

To directly compare the effect of cue relevance on target detection between groups, we used the data from invalid cueing conditions to compute a relevance index as follows: $\mathrm{RI}=$ $\left(\mathrm{RT}_{\text {relevant }}-\mathrm{RT}_{\text {irrelevant }}\right) /\left(\mathrm{RT}_{\text {relevant }}+\mathrm{RT}_{\text {irrelevant }}\right)$. The relevance index is independent of baseline processing time and reflects the extent to which the speed of target detection depends on cue relevance. A positive index indicates a processing cost, whereas a negative index reflects a processing advantage of targets following relevant compared with irrelevant cues. Relevance indices for left targets were strongly positive for all patients in the relevance neglect group, but close to zero in the nonrelevance neglect group (Fig. 3a). In all groups, average relevance indices only slightly differed from zero, except for left targets in the relevance neglect patients (Fig. $3 b$ ). An ANOVA with group and target position as factors revealed a significant group effect $\left(F_{(3,37)}=17.7, p<\right.$ $0.0001)$ and a significant interaction $\left(F_{(3,37)}=10.91, p<0.0001\right)$, indicating that relevance neglect patients had larger relevance indices for left targets than all other groups (all $p<0.0001$ ). These patients were also the only group with a significant asymmetry between indices for left and right targets $(p<0.001)$. Thus, in contrast to all other groups, attention of the relevance neglect group was strongly captured by right-sided task-relevant stimuli.

When interpreting these group comparisons, one should be aware that some statistical differences between the two neglect subgroups can be expected based on the a priori assignment of patients to the relevance or nonrelevance group. However, it is crucial for the interpretation of anatomical differences presented in the next section that the only difference between the two neglect groups is related to the effect of task relevance of ipsilesional

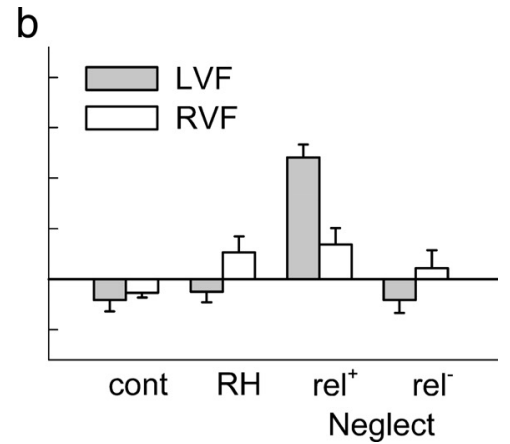

Patient cues, and that no other fundamental differences in their response pattern exist.

\section{Lesion comparisons}

Behavioral data distinguished two groups of neglect patients whose reorienting of attention toward contralesional targets was differently affected by the relevance of the cue. We next examined whether the effect of cue relevance or its absence was associated with damage to specific regions of the right-hemispheric attention network. To make a comparison with previous studies, we first searched to identify those areas in which damage was correlated with spatial neglect regardless of neglect subgroup by subtracting from the lesion overlaps of all 20 neglect patients (Fig. $4 a$ ) the lesions of the RH control patients (Fig. $4 b$ ). Note that the significance of the comparison between neglect patients and $\mathrm{RH}$ controls is necessarily limited due to the difference in lesion volume (Table 2), and that the unique purpose of this subtraction analysis is to verify whether our sample of neglect patients is comparable to previously studied patients when the same analysis is applied. The lesion overlap analysis identified three regions that were associated with spatial neglect (Fig. $4 a$ ), as follows: the right periventricular white matter (MNI voxel-coordinates: $30,-22$, 22; damaged in 13 of 20 neglect patients), the white matter beneath the middle frontal gyrus (coordinates: 27, 3, 30; damaged in 11 neglect patients), and the white matter beneath the angular gyrus (coordinates: 44, -46, 32; damaged in 12 neglect patients). The latter region was damaged in none of the control patients and was therefore identified as the maximal difference $(60 \%)$ between groups in the subtraction analysis (Fig. 4c). These findings are highly consistent with previous studies examining the anatomy of spatial neglect (Vallar and Perani, 1986; Mort et al., 2003; Golay et al., 2008); the lesions of our patients were thus representative of the common lesion sites in spatial neglect.

We next generated distinct lesion overlaps for each of the two neglect groups. These overlaps revealed that the relevance neglect group had cortical and subcortical damage in the inferior perisylvian region (Fig. 5a), whereas the nonrelevance group had more dorsal lesions affecting the frontoparietal cortex and the white matter of the frontal lobe (Fig. $5 b$ ). The group subtraction (Fig. $5 c$ ) revealed most frequent damage in the anterior limb of the internal capsule (difference, 60\%; voxel coordinates, $22,8,15$ ) in the relevance neglect group and most frequent damage in the white matter beneath the superior frontal gyrus (difference, 90\%; voxel coordinates, $24,6,40)$ in the nonrelevance neglect group. 


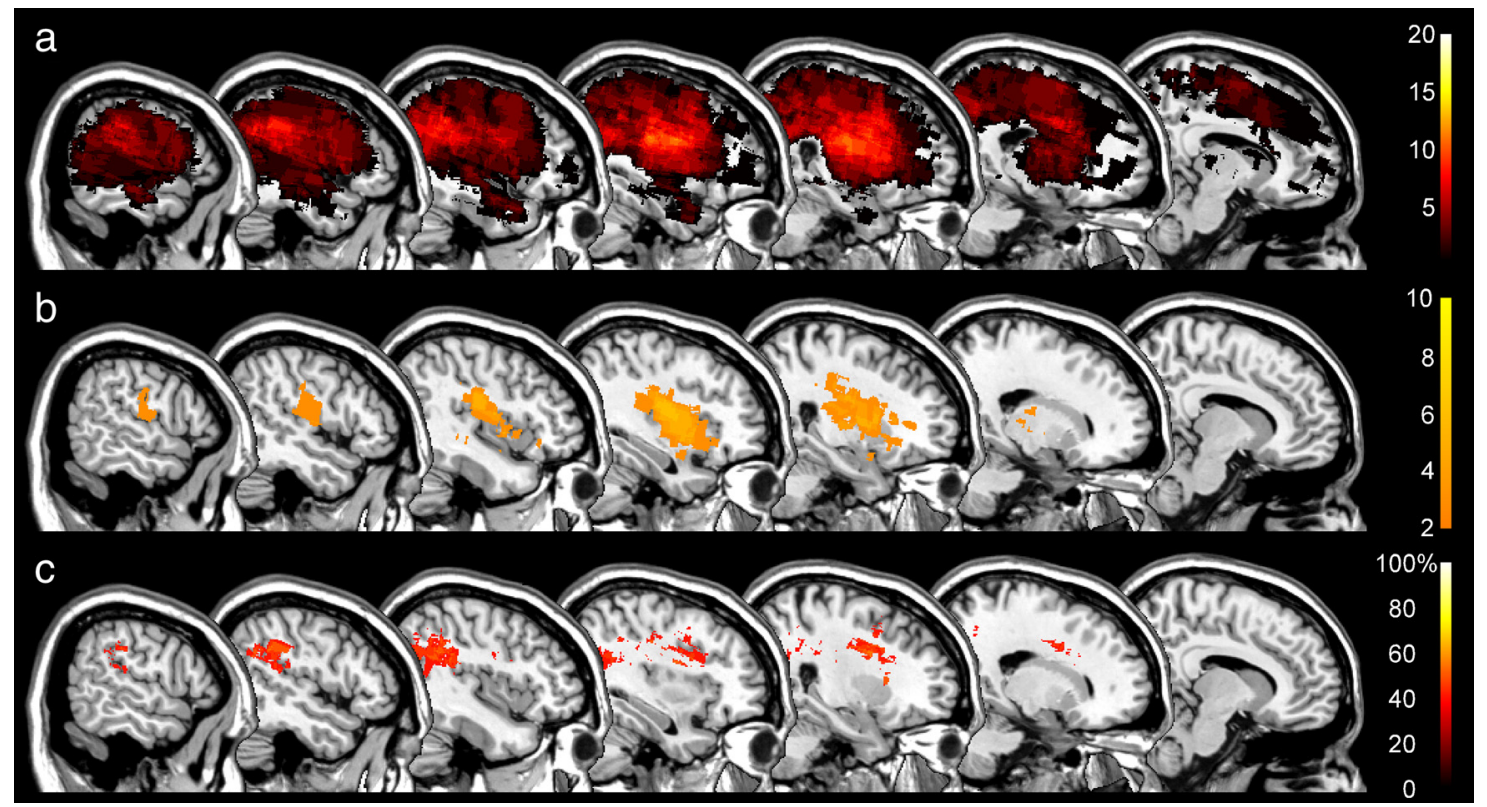

Figure 4. Anatomy of neglect and nonneglect patients. $\boldsymbol{a}, \boldsymbol{b}$, Color-coded overlap images of the lesions of all 20 neglect patients $(\boldsymbol{a})$ and right-hemisphere controls ( $\boldsymbol{b}$ ) projected on sagittal sections of the MNI template brain with $x$-coordinates increasing from right $(x=10)$ to left $(x=58)$. The color scales indicate the increasing number of overlapping lesions starting at $n=2$. $c$, Subtraction plot showing regions of at least $40 \%$ greater relative prevalence of damage in the neglect group than the right-hemisphere controls. The color scale indicates the percentage of damage to specific voxels associated with spatial neglect.

Table 2. Lesion characteristics of RH-damaged control patients and the two neglect groups (mean \pm SEM)

\begin{tabular}{|c|c|c|c|c|c|c|}
\hline & Volume $\left(\mathrm{cm}^{3}\right)$ & Grey matter (\%) & Center of mass $(x / y / z)$ & $\mathrm{BA} 6\left(\mathrm{~cm}^{3}\right)$ & $\mathrm{BA} 8\left(\mathrm{~cm}^{3}\right)$ & BA $9\left(\mathrm{~cm}^{3}\right)$ \\
\hline RH control & $24.4( \pm 7.4)$ & $35.2( \pm 6.4)$ & $123 / 110 / 86$ & $1.23( \pm 0.95)$ & $0.07( \pm 0.07)$ & $0.13( \pm 0.13)$ \\
\hline Relevance neglect & $100.2( \pm 23.4)$ & $42.5( \pm 6.2)$ & $124 / 109 / 84$ & $2.03( \pm 1.4)$ & $0.91( \pm 0.9)$ & $1.41( \pm 1.4)$ \\
\hline Nonrelevance neglect & $134.7( \pm 35)$ & $47.8( \pm 5.7)$ & $124 / 111 / 106$ & $17.31( \pm 4.3)$ & $2.24( \pm 0.7)$ & $3.14( \pm 1.1)$ \\
\hline
\end{tabular}

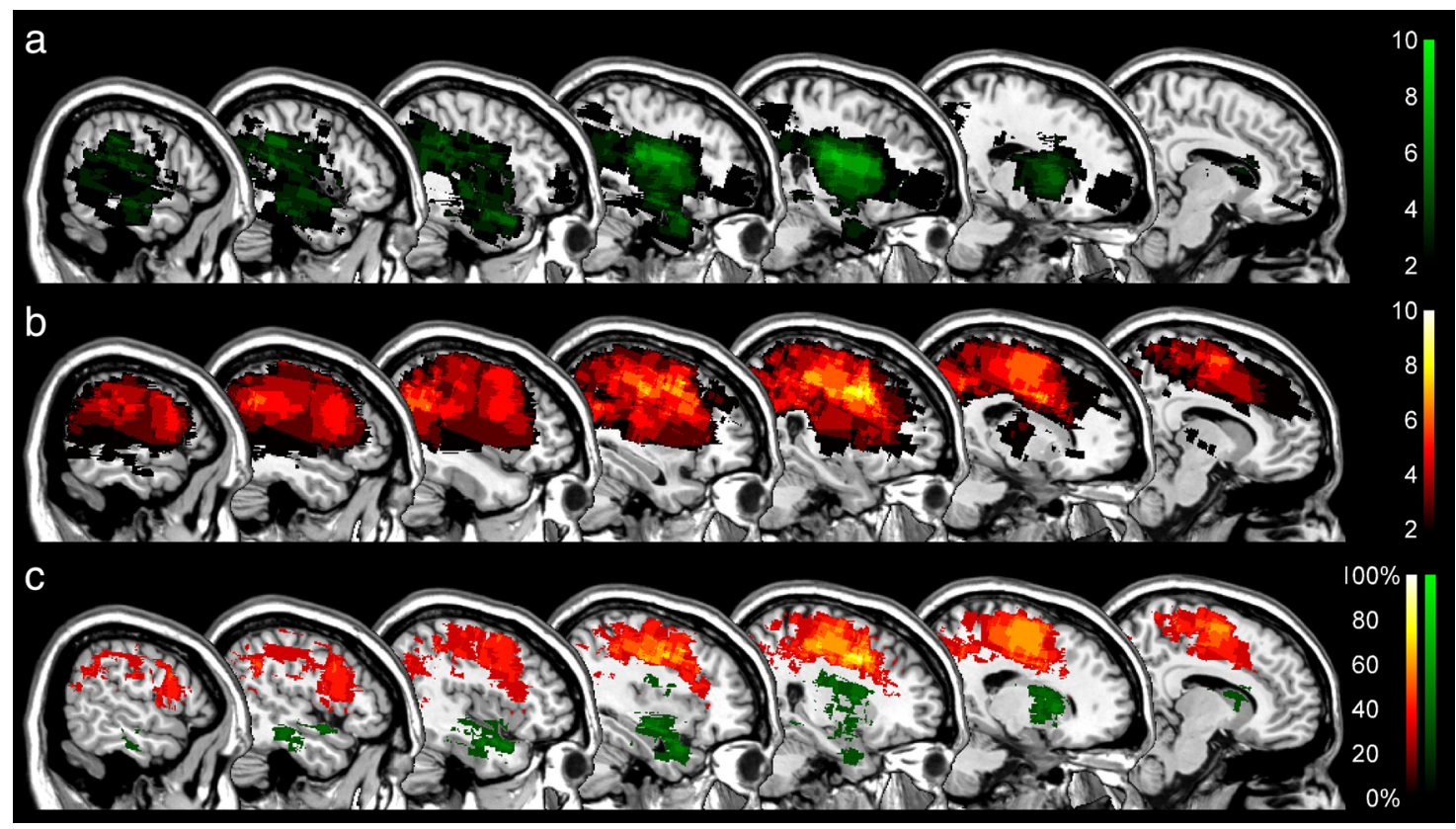

Figure 5. Anatomy of the effect of relevant cues in neglect. $\boldsymbol{a}$, Lesion overlap of neglect patients with a relevance effect. $\boldsymbol{b}$, Lesion overlap of neglect patients without a relevance effect. $\boldsymbol{c}$, Subtraction plot showing regions associated with presence (green) and absence (red) of a relevance effect in neglect. The color scales indicate the frequency of involvement, between 0\% (dark) and 100\% (light).

These anatomical differences were also evident in the position of the center of mass of individual lesions, which lay significantly more dorsally in the nonrelevance than the relevance neglect group (Mann-Whitney test, $p<0.01$ ) (Table 2). In contrast, the differences between groups were not related to overall lesion volume or the percentage of gray or white matter involvement (Table 2).

To further examine the extent of cortical damage within specific areas of the dorsal attention network, we segmented the 


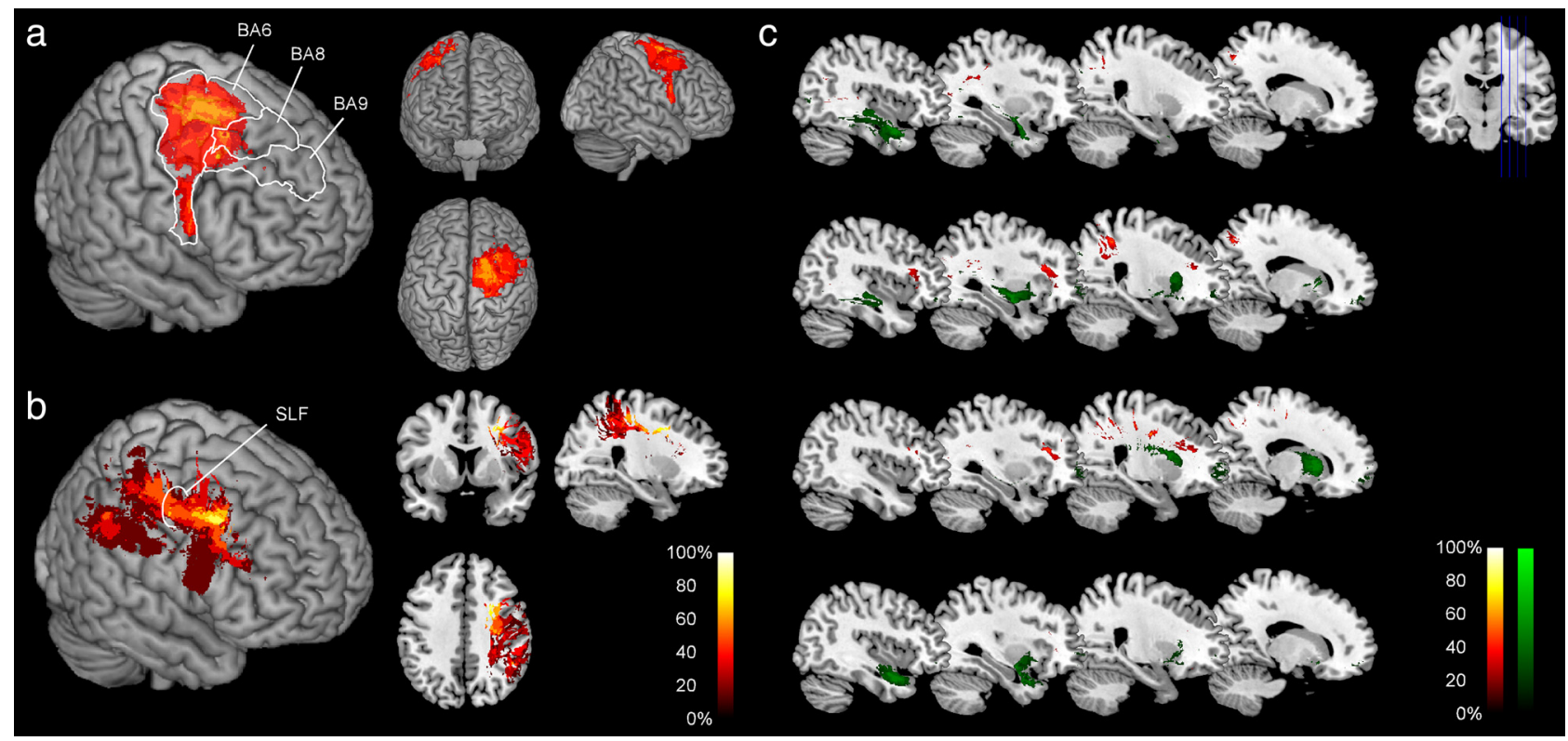

Figure 6. Relevance effect and the dorsal attention network. $\boldsymbol{a}$, Subtraction plot showing Brodmann areas where damage was at least $20 \%$ more frequent in the nonrelevance than the relevance neglect group. $\boldsymbol{b}$, Subtraction plot showing components of the SLF where damage was at least $20 \%$ more frequent in the nonrelevance than the relevance neglect group. $\boldsymbol{c}$, Subtraction plots showing components of specific fiber tracts where damage was at least $20 \%$ more frequent in the nonrelevance than the relevance neglect group (red) or more frequent in the relevance than the nonrelevance neglect group (green). From top to bottom, Inferior longitudinal fasciculus, inferior fronto-occipital fasciculus, anterior thalamic radiation, and uncinate fasciculus. Sagittal sections with $x$-coordinates increasing from right $(x=18)$ to left $(x=42)$ are shown.

lesion of each participant using a brain template with specified Brodmann areas (BA). The comparison of the volume of damage to these areas revealed greater involvement of BA 6, 8, and 9 (Mann-Whitney test, all $p<0.05$ ) in the nonrelevance than the relevance group (Table 2). Subtracting lesions of the relevance group from the nonrelevance group showed that the main difference between groups at the cortical level was situated in BA 6 (Fig. 6a).

Several studies have suggested that frontoparietal disconnection by damage to associative fiber tracts may be more predictive of the occurrence of neglect than cortical damage (Gaffan and Hornak, 1997; Doricchi and Tomaiuolo, 2003; Thiebaut de Schotten et al., 2005). As the maximal lesion overlap of the nonrelevance group was subcortical, we examined the involvement of intrahemispheric fiber tracts by segmenting the lesions of the patients with fiber tracts from a stereotaxic atlas of white matter anatomy based on diffusion-tensor imaging of normal brains (Mori et al., 2008). If damage in nonrelevance neglect patients affected the dorsal attention network, we would expect that lesions of these patients overlap with the superior longitudinal fasciculus (SLF), which is the main fiber pathway that connects the dorsal premotor and dorsolateral prefrontal cortex with the parietal lobe (Schmahmann and Pandya, 2006). Each patient VOI was segmented using information about the localization of the SLF, and voxel-based subtractions between the nonrelevance and the relevance groups were then performed using these segmented VOIs. To examine whether intrahemispheric tracts other than the SLF were predictors of performance, we performed the same analysis with four other fiber tracts, as follows: the inferior longitudinal fasciculus, the inferior fronto-occipital fasciculus, the anterior thalamic radiation, and the uncinate fasciculus. The SLF was more frequently damaged in the nonrelevance than the relevance group (Fig. 6b), with maximal difference in its rostral part (coordinates, 24, 0, 38; damaged in 9 of 10 patients of the nonrelevance and none of the 10 patients of the relevance group). The only patient of the nonrelevance group in whom the anterior SLF was spared had damage to the inferior parietal lobule including the angular and supramarginal gyrus, which are the posterior projection sites of parts of the SLF (Schmahmann and Pandya, 2006). Damage to the SLF was the critical predictor of whether a patient belonged to the nonrelevance group as the other, more ventrally located fiber tracts were almost completely spared in the nonrelevance group (Fig. 6c).

To provide a statistical test of these results, we performed two independent VLSM analyses that both used the relevance index as predictor variable. In the first analysis, the relevance index was used as a binomial measure to define the groups ( 1 = relevanceneglect group, $0=$ nonrelevance neglect group), and the nonparametric Liebermeister measure was used to compute significant voxels. In the second analysis, individual relevance indices were entered as continuous variable and the nonparametric Brunner-Munzel test was used to perform comparisons on a voxel-by-voxel basis. Note that this latter analysis is fully independent of a priori attribution of patients to one of the two groups. Figure 7 shows brain areas that were significant predictors of the absence of a relevance effect in neglect patients using the binomial test and the Brunner-Munzel test for continuous measures (FWE-corrected $\alpha$-level of $p<0.05$ ). The binomial test identified a dorsal frontal region anterior to the precentral sulcus reaching into the white matter beneath the superior frontal gyrus as critical for the absence of a relevance effect. The most anterior voxels, lying in the white matter beneath the superior frontal sulcus (coordinates, 24, 0, 41), were also significant predictors of the absence of a relevance effect when the more conservative Brunner-Munzel test was used. This region is identical to the maximal overlap of lesions of patients belonging to the nonrelevance neglect group. The VLSM analyses thus statistically confirmed the group comparisons based on lesion overlap counts. 


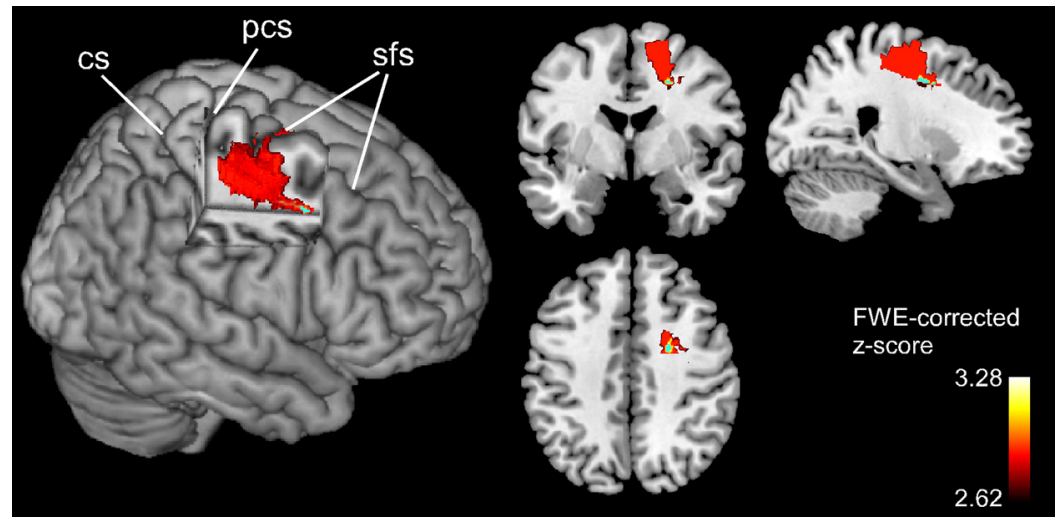

Figure 7. Results of lesion-symptom mapping analyses. Voxels that survived correction for multiple comparisons using the Liebermeister measure for binomial data are shown in red and yellow. Voxels with damage that was a significant negative predictor of the relevance effect (Brunner-Munzel test for continuous measures) are shown in light blue. CS, Central sulcus; PCS: precentral sulcus; SFS: superior frontal sulcus.

\section{Discussion}

The present findings provide lesion evidence for a modulatory role of the dorsal attention network on the orienting of attention in spatial neglect. The cueing paradigm used in this study enables us to distinguish between facilitation by perceptually similar cues and effects related to current action goals. In the relevant identical condition, cues were identical to the upcoming target. Any effect of these cues may therefore reflect perceptual facilitation, capture of attention, or a combination of these factors. In contrast, facilitatory or inhibitory effects of relevant different cues can only be attributed to their behavioral significance, as these are perceptually dissimilar to the target.

Regardless of cue validity, both control groups exhibited faster reactions with relevant identical cues compared with relevant different and irrelevant cues, a pattern suggesting facilitation by perceptually similar cues. Contrasting with the results of previous studies showing slower reactions following invalid cues (Eriksen and Hoffman, 1972; Posner, 1980), the control groups showed no difference in performance following valid and invalid cues (i.e., no validity effect). However, facilitation of target detection by valid cues is a function of time between cue and target onset; in healthy participants, facilitation is only observed for several hundred milliseconds following cue onset, whereupon the effect of valid cues becomes inhibitory (Posner et al., 1985). In the present study, cue and target were separated by $500 \mathrm{~ms}$, which may explain the absence of a validity effect in the control groups. However, this interval was insufficient to abolish the characteristic impairment of attentional disengagement from ipsilesional cues observed in the two neglect groups.

Previous studies observed impaired reorienting of attention in neglect patients following presentation of ipsilesional cues for stimuli as various as colored forms, letters, whole words, when the cue was a word and the target a colored circle (Ptak and Golay, 2006; Ptak and Schnider, 2006), and for cue-target pairings within or across modalities (Farah et al., 1989; Golay et al., 2005; Ptak and Schnider, 2005). Thus, the critical variable determining whether a cue captures attention of neglect patients is not its similarity to the target, but its relevance for the current task. The finding that, in the present study, relevant cues gave rise to very similar performance, be they visually similar to the target or not, also suggests that the influence of perceptual interference on reorienting of attention was minor compared with the effect of cue relevance. Further, the fact that both neglect groups had similar rates of false-positive responses argues against an explanation of the differences in relevance encoding in terms of different degrees of response inhibition failure. Finally, the question arises whether the results might be explained by a prolonged attentional blink, a phenomenon characterized by the failure to detect the second of two targets presented in rapid sequence among distracters (Husain et al., 1997). However, in contrast to paradigms that typically evoke the attentional blink (Martens and Wyble, 2010), participants were asked to disregard the cue and to react uniquely to the target. In addition, there was no evidence for an attentional blink in the data of control participants or for ipsilesional and validly cued targets in neglect patients, which argues against this account.

Our findings resolve several issues concerning the functional and anatomical characteristics of spatial neglect and the involvement of the dorsal attention network in this disorder. First, they reconcile results of studies showing that attention of neglect patients shifts to right-sided stimuli regardless of their relevance for the present task (Gainotti et al., 1991; Snow and Mattingley, 2005) and the contrasting observation that capture of attention critically depends on task constraints (Ptak et al., 2002; Ptak and Schnider, 2006). According to the present findings, both views are correct, but each of them only relates to a subgroup of neglect patients with distinct lesion anatomy; patients with a preserved dorsal attention network may have a strong tendency to orient attention toward stimuli of high relevance, whereas attention of patients with damage or disconnection of this network is captured indifferently by any stimulus. The present results thus support the view that, to better understand the functional complexity of the neglect syndrome, its anatomy should be studied by differentiating neglect subgroups on the basis of distinct patterns of performance in specific tasks (Hillis et al., 2005; Ptak and Valenza, 2005).

Second, the observation of a functional dissociation between neglect patients with and patients without damage affecting the dorsal attention network contributes to the recent controversy regarding the anatomy of spatial neglect. Lesion overlap studies located the critical region responsible for spatial neglect in the inferior parietal cortex (Vallar and Perani, 1986; Mort et al., 2003; Golay et al., 2008), whereas Karnath et al. (2004) attributed a similar role to the central sectors of the superior temporal gyrus. In contrast, Doricchi and Tomaiuolo (2003) emphasized the role of frontoparietal disconnection as possible anatomical basis of spatial neglect. This proposal has recently received support from a study of functional connectivity using measures of coherent fluctuations of functional MRI signals, which has revealed that parietal damage in neglect is accompanied by impaired connectivity in the dorsal attention network (He et al., 2007). Moreover, a study using intraoperative stimulation in patients undergoing surgery for a parietal tumor found that functional inhibition of the SLF generates a neglect-like pattern in the line bisection task (Thiebaut de Schotten et al., 2005). These findings suggest that intrahemispheric frontoparietal disconnection is crucial for the occurrence of spatial neglect (Thiebaut de Schotten et al., 2005; Bartolomeo et al., 2007).

The SLF is the main association fiber tract that links the parietal and frontal cortex (Makris et al., 2005; Schmahmann and Pandya, 2006). Based on studies of the monkey brain, three sub- 
divisions have been identified that connect the superior parietal region with the supplementary motor area (SLF I), the inferior parietal cortex with dorsal frontal areas 6, 8, 9, and 46 (SLF II), and the supramarginal gyrus with ventral premotor cortex (mainly the ventral part of area 6 and area 44) (SLF III) (Petrides and Pandya, 2002; Rushworth et al., 2006). The main component of the SLF (SLF II) connects the FEF [which in humans is located at the intersection between the precentral and superior frontal sulcus, i.e., BA 6 (Paus, 1996)], with parietal regions around the angular gyrus involved in spatial processing (Petrides and Pandya, 2002). The connection pattern of the SLF and the finding that cortical damage of nonrelevance neglect patients was centered on BA 6 suggest that the component of this fiber tract that is critical for modulation of attention by task relevance is SLF II.

The third contribution of this study is a better understanding of the functional role of the dorsal attention network. The FEF and PPC are strongly activated by attention-directing cues (e.g., a central arrow) or when subjects focus attention on a particular stimulus feature (Corbetta et al., 2000; Hopfinger et al., 2000), suggesting a preferential role of the dorsal attention network for voluntary orienting. Our findings identify an additional role for the FEF and its parietal connections. Whether a cue was presented at the same location as the upcoming target or at the opposite location did not influence performance of the two control groups. In contrast, though they did not predict the presence or location of the target, right peripheral cues strongly captured attention in neglect patients. This finding suggests that cues presented in the right hemifield engaged reflexive mechanisms of attention in neglect patients, but not in healthy and braindamaged participants without spatial neglect (Bartolomeo et al., 2001). However, at cue-target intervals shorter than those used in the present study, even healthy participants show a reflexive facilitation by valid compared with invalid cues (Posner, 1980). Thus, one functional consequence of spatial neglect is to prolong the time interval during which abrupt onsets increase the saliency of peripheral cues and trigger reflexive mechanisms of attention. Consequently, the behavioral dissociation between patients with intact and with damaged dorsal attention networks suggests that this network modulates reflexive capture of attention through a mechanism that temporarily amplifies the saliency of behaviorally relevant events and attenuates the saliency of irrelevant events. Evidence that attention enhances or inhibits sensory processing has been provided by psychophysical (Blaser et al., 1999), electrophysiological (Hillyard et al., 1998; Ptak et al., 2010), and neurophysiological studies (Fecteau and Munoz, 2006). Interestingly, visuomotor neurons in the monkey FEF have response characteristics that are analogous to the response pattern of relevance neglect patients. These neurons show increased responses to distracters that share a feature with the target of a saccade than to neutral distracters, and the magnitude of their response reflects the current behavioral significance of the stimulus (Bichot and Schall, 1999).

In summary, in agreement with findings from monkey neurophysiology and functional imaging of healthy participants, our findings show that modulation of spatial processing critically depends on the integrity of the dorsal attention network and that the preservation of the latter spatial neglect leads to an exaggerated spatial bias favoring task-relevant stimuli.

\section{References}

Bartolomeo P, Siéroff E, Decaix C, Chokron S (2001) Modulating the attentional bias in unilateral neglect: the effects of the strategic set. Exp Brain Res 137:432-444.
Bartolomeo P, Thiebaut de Schotten M, Doricchi F (2007) Left unilateral neglect as a disconnection syndrome. Cereb Cortex 17:2479-2490.

Bates E, Wilson SM, Saygin AP, Dick F, Sereno MI, Knight RT, Dronkers NF (2003) Voxel-based lesion-symptom mapping. Nat Neurosci 6:448-450.

Bichot NP, Schall JD (1999) Effects of similarity and history on neural mechanisms of visual selection. Nat Neurosci 2:549-554.

Blaser E, Sperling G, Lu ZL (1999) Measuring the amplification of attention. Proc Natl Acad Sci U S A 96:11681-11686.

Bruce CJ, Goldberg ME (1985) Primate frontal eye fields. I. Single neurons discharging before saccades. J Neurophysiol 53:603-635.

Buschman TJ, Miller EK (2007) Top-down versus bottom-up control of attention in the prefrontal and posterior parietal cortices. Science 315:1860-1862.

Constantinidis C, Steinmetz MA (2001) Neuronal responses in area 7a to multiple-stimulus displays. I. Neurons encode the location of the salient stimulus. Cereb Cortex 11:581-591.

Corbetta M, Shulman GL (2002) Control of goal-directed and stimulusdriven attention in the brain. Nat Rev Neurosci 3:201-215.

Corbetta M, Kincade JM, Ollinger JM, McAvoy MP, Shulman GL (2000) Voluntary orienting is dissociated from target detection in human posterior parietal cortex. Nat Neurosci 3:292-297.

Doricchi F, Tomaiuolo F (2003) The anatomy of neglect without hemianopia: a key role for parietal-frontal disconnection? Neuroreport 14:2239-2243.

Egeth HE, Yantis S (1997) Visual attention: control, representation, and time course. Annu Rev Psychol 48:269-297.

Eriksen CW, Hoffman JE (1972) Temporal and spatial characteristics of selective encoding from visual displays. Percept Psychophys 12:201-204.

Farah MJ, Wong AB, Monheit MA, Morrow LA (1989) Parietal lobe mechanisms of spatial attention: modality-specific or supramodal? Neuropsychologia 27:461-470.

Fecteau JH, Munoz DP (2006) Salience, relevance, and firing: a priority map for target selection. Trends Cogn Sci 10:382-390.

Folk CL, Remington RW, Johnston JC (1992) Involuntary covert orienting is contingent on attentional control settings. J Exp Psychol Hum Percept Perform 18:1030-1044.

Gaffan D, Hornak J (1997) Visual neglect in the monkey. Brain 120:1647-1657.

Gainotti G, D’Erme P, Bartolomeo P (1991) Early orientation of attention toward the half space ipsilateral to the lesion in patients with unilateral brain damage. J Neurol Neurosurg Psychiatry 54:1082-1089.

Gauthier L, Dehaut F, Joanette Y (1989) The Bells test: a quantative and qualitative test for visual neglect. Int J Clin Neuropsychol 11:49-54.

Gitelman DR, Nobre AC, Parrish TB, LaBar KS, Kim YH, Meyer JR, Mesulam M (1999) A large-scale distributed network for covert spatial attention. Brain 122:1093-1106.

Golay L, Hauert CA, Greber C, Schnider A, Ptak R (2005) Dynamic modulation of visual detection by auditory cues in spatial neglect. Neuropsychologia 43:1258-1265.

Golay L, Schnider A, Ptak R (2008) Cortical and subcortical anatomy of chronic spatial neglect following vascular damage. Behav Brain Funct 4:43.

Gottlieb JP, Kusunoki M, Goldberg ME (1998) The representation of visual salience in monkey parietal cortex. Nature 391:481-484.

Halligan PW, Fink GR, Marshall JC, Vallar G (2003) Spatial cognition: evidence from visual neglect. Trends Cogn Sci 7:125-133.

He BJ, Snyder AZ, Vincent JL, Epstein A, Shulman GL, Corbetta M (2007) Breakdown of functional connectivity in frontoparietal networks underlies behavioral deficits in spatial neglect. Neuron 53:905-918.

Heilman KM, Watson RT, Valenstein E (1993) Neglect and related disorders. In: Clinical neuropsychology (Heilman KM, Valenstein E, eds), pp 279-336. New York: Oxford UP.

Hillis AE, Newhart M, Heidler J, Barker PB, Herskovits EH, Degaonkar M (2005) Anatomy of spatial attention: insights from perfusion imaging and hemispatial neglect in acute stroke. J Neurosci 25:3161-3167.

Hillyard SA, Vogel EK, Luck SJ (1998) Sensory gain control (amplification) as a mechanism of selective attention: electrophysiological and neuroimaging evidence. Philos Trans R Soc Lond B Biol Sci 353:1257-1270.

Hopfinger JB, Buonocore MH, Mangun GR (2000) The neural mechanisms of top-down attentional control. Nat Neurosci 3:284-291.

Husain M, Shapiro K, Martin J, Kennard C (1997) Abnormal temporal dynamics of visual attention in spatial neglect patients. Nature 385:154-156. 
Indovina I, Macaluso E (2007) Dissociation of stimulus relevance and saliency factors during shifts of visuospatial attention. Cereb Cortex 17:1701-1711.

Karnath HO, Fruhmann Berger M, Küker W, Rorden C (2004) The anatomy of spatial neglect based on voxelwise statistical analysis: a study of 140 patients. Cereb Cortex 14:1164-1172.

Kirchner H, Barbeau EJ, Thorpe SJ, Régis J, Liégeois-Chauvel C (2009) Ultra-rapid sensory responses in the human frontal eye field region. J Neurosci 29:7599-7606.

Losier BJ, Klein RM (2001) A review of the evidence for a disengage deficit following parietal lobe damage. Neurosci Biobehav Rev 25:1-13.

Makris N, Kennedy DN, McInerney S, Sorensen AG, Wang R, Caviness VS Jr, Pandya DN (2005) Segmentation of subcomponents within the superior longitudinal fascicle in humans: a quantitative, in vivo, DT-MRI study. Cereb Cortex 15:854-869.

Martens S, Wyble B (2010) The attentional blink: past, present, and future of a blind spot in perceptual awareness. Neurosci Biobehav Rev 34:947-957.

Mori S, Oishi K, Jiang H, Jiang L, Li X, Akhter K, Hua K, Faria AV, Mahmood A, Woods R, Toga AW, Pike GB, Neto PR, Evans A, Zhang J, Huang H, Miller MI, van Zijl P, Mazziotta J (2008) Stereotaxic white matter atlas based on diffusion tensor imaging in an ICBM template. Neuroimage 40:570-582.

Morrow LA, Ratcliff G (1988) The disengagement of covert attention and the neglect syndrome. Psychobiology 16:261-269.

Mort DJ, Malhotra P, Mannan SK, Rorden C, Pambakian A, Kennard C, Husain M (2003) The anatomy of visual neglect. Brain 126:1986-1997.

Paus T (1996) Location and function of the human frontal eye-field: a selective review. Neuropsychologia 34:475-483.

Petrides M, Pandya DN (2002) Association pathways of the prefrontal cortex and functional observations. In: Principles of frontal lobe function (Stuss DT, Knight RT, eds), pp 31-50. New York: Oxford UP.

Posner MI (1980) Orienting of attention. Q J Exp Psychol 32:3-25.

Posner MI, Walker JA, Friedrich FJ, Rafal RD (1984) Effects of parietal injury on covert orienting of attention. J Neurosci 4:1863-1874.

Posner MI, Rafal RD, Choate LS, Vaughan J (1985) Inhibition of return: neural basis and function. Cogn Neuropsychol 2:211-228.

Ptak R, Golay L (2006) Temporal dynamics of attentional control settings in patients with spatial neglect. Brain Res 1092:190-197.

Ptak R, Schnider A (2005) Visual extinction of similar and dissimilar stimuli: evidence for level-dependent attentional competition. Cogn Neuropsychol 22:111-127.
Ptak R, Schnider A (2006) Reflexive orienting in spatial neglect is biased towards behaviourally salient stimuli. Cereb Cortex 16:337-345.

Ptak R, Valenza N (2005) The inferior temporal lobe mediates distracterresistant visual search of patients with spatial neglect. J Cogn Neurosci 17:788-799.

Ptak R, Valenza N, Schnider A (2002) Expectation-based attentional modulation of visual extinction in spatial neglect. Neuropsychologia 40:2199-2205.

Ptak R, Schnider A, Golay L, Müri R (2007) A non-spatial bias favouring fixated stimuli revealed in patients with spatial neglect. Brain 130:3211-3222.

Ptak R, Camen C, Morand S, Schnider A (2010) Early event-related cortical activity originating in the frontal eye fields and inferior parietal lobe predicts the occurrence of correct and error saccades. Hum Brain Mapp. Advance online publication. Retrieved June 28, 2010. doi: 10.1002/hbm. 21025 .

Rorden C, Karnath HO, Bonilha L (2007) Improving lesion-symptom mapping. J Cogn Neurosci 19:1081-1088.

Rushworth MF, Behrens TE, Johansen-Berg H (2006) Connection patterns distinguish 3 regions of human parietal cortex. Cereb Cortex 16:1418-1430.

Schall JD, Thompson KG (1999) Neural selection and control of visually guided eye movements. Annu Rev Neurosci 22:241-259.

Schenkenberg T, Bradford DC, Ajax ET (1980) Line bisection and unilateral visual neglect in patients with neurologic impairment. Neurology 30:509-517.

Schmahmann JD, Pandya DN (2006) Fiber pathways of the brain. Oxford: Oxford UP.

Snow JC, Mattingley JB (2006) Goal-driven selective attention in patients with right hemisphere lesions: how intact is the ipsilesional field? Brain 129:168-181.

Thiebaut de Schotten M, Urbanski M, Duffau H, Volle E, Lévy R, Dubois B, Bartolomeo P (2005) Direct evidence for a parietal-frontal pathway subserving spatial awareness in humans. Science 309:2226-2228.

Vallar G, Perani D (1986) The anatomy of unilateral neglect after righthemisphere stroke lesions: a clinical/CT-scan correlation study in man. Neuropsychologia 24:609-622.

Wilson B, Cockburn J, Halligan P (1987) Behavioural inattention test. Bury St Edmunds: Thames Valley Test Company.

Yantis S, Jonides J (1990) Abrupt visual onsets and selective attention: voluntary versus automatic allocation. J Exp Psychol Hum Percept Perform $16: 121-134$. 